\title{
Free Convective MHD Flow Past a Vertical Cone with Variable Heat and Mass Flux
}

\author{
J. Prakash, ${ }^{1}$ S. Gouse Mohiddin, ${ }^{2}$ and S. Vijaya Kumar Varma ${ }^{3}$ \\ ${ }^{1}$ Department of Mathematics, University of Botswana, Private Bag 0022, Gaborone, Botswana \\ ${ }^{2}$ Department of Mathematics, Madanapalle Institute of Technology \& Science, Madanapalle 517325, Andhra Pradesh, India \\ ${ }^{3}$ Department of Mathematics, Sri Venkateswara University, Tirupati 517502, Andhra Pradesh, India
}

Correspondence should be addressed to J. Prakash; prakashj@mopipi.ub.bw

Received 23 April 2013; Revised 17 September 2013; Accepted 24 September 2013

Academic Editor: Mohy S. Mansour

Copyright (c) 2013 J. Prakash et al. This is an open access article distributed under the Creative Commons Attribution License, which permits unrestricted use, distribution, and reproduction in any medium, provided the original work is properly cited.

\begin{abstract}
A numerical study of buoyancy-driven unsteady natural convection boundary layer flow past a vertical cone embedded in a nonDarcian isotropic porous regime with transverse magnetic field applied normal to the surface is considered. The heat and mass flux at the surface of the cone is modeled as a power law according to $q_{w}(x)=x^{m}$ and $q_{w}^{*}(x)=x^{m}$, respectively, where $x$ denotes the coordinate along the slant face of the cone. Both Darcian drag and Forchheimer quadratic porous impedance are incorporated into the two-dimensional viscous flow model. The transient boundary layer equations are then nondimensionalized and solved by the Crank-Nicolson implicit difference method. The velocity, temperature, and concentration fields have been studied for the effect of Grashof number, Darcy number, Forchheimer number, Prandtl number, surface heat flux power-law exponent $(m)$, surface mass flux power-law exponent $(n)$, Schmidt number, buoyancy ratio parameter, and semivertical angle of the cone. Present results for selected variables for the purely fluid regime are compared with the published results and are found to be in excellent agreement. The local skin friction, Nusselt number, and Sherwood number are also analyzed graphically. The study finds important applications in geophysical heat transfer, industrial manufacturing processes, and hybrid solar energy systems.
\end{abstract}

\section{Introduction}

Combined heat and mass transfer in fluid-saturated porous media finds applications in a variety of engineering processes such as heat exchanger devices, petroleum reservoirs, chemical catalytic reactors and processes, and others. A thorough discussion of these and other applications is available in the monographs $[1,2]$. Comprehensive reviews of the much of the work communicated in porous media transport phenomena have been presented in [3,4]. Most studies dealing with porous media have employed the Darcy law. However, for high velocity flow situations, the Darcy law is inapplicable, since it does not account for inertial effects in the porous medium. Such flows can arise, for example, in the near-wellbore region of high capacity gas and condensate petroleum reservoirs and also in highly porous filtration systems under high blowing rates. The most popular approach for simulating high-velocity transport in porous media is the Darcy-Forchheimer drag force model. This adds a second-order (quadratic) drag force to the momentum transport equation. This term is related to the geometrical features of the porous medium and is independent of viscosity. A seminal study discussing the influence of Forchheimer inertial effects in porous media convection is presented by Vafai and Tien [5]. The mixed convective boundary layer flow from a vertical surface in a fluid-saturated non-Darcian porous medium including Forchheimer inertial effects is studied by C.-H. Chen and C.-K. Chen [6] and Chen et al. [7]. Thermal convection boundary layer flow with buoyancy and suction/blowing effects from a cone with nonuniform surface temperature is studied by Hossain and Paul [8]. The study is extended by Hossain and Paul [9] by considering nonuniform surface heat flux, both studies employing numerical methods. Chamkha et al. [10] studied the double-diffusive convection heat and mass transfer over a cone (and wedge) in DarcyForchheimer porous media. Magnetohydrodynamic (MHD) 
flow and heat transfer is of considerable interest because it can occur in many geothermal, geophysical, technological, and engineering applications such as nuclear reactors and others. The geothermal gases are electrically conducting and are affected by the presence of a magnetic field. Vajravelu and Nayfeh [11] studied hydromagnetic convection from a cone and a wedge with variable surface temperature and internal heat generation or absorption. Thus far the transient thermal convection flow over a cone in Darcy-Forchheimer porous media has not been studied in the literature despite important applications in geothermics, geophysics, and materials processing.

\section{Mathematical Model}

An axisymmetric unsteady natural convection boundary layer flow past a vertical cone with transverse magnetic field applied normal to the surface with variable heat and mass flux in a Darcy-Forchheimer fluid saturated porous medium in a cartesian $(x, y)$ coordinate system is formulated mathematically in this section. Initially, it is assumed that the cone surface and the surrounding fluid which are at rest possess the same temperature $T_{\infty}^{\prime}$ and concentration level $C_{\infty}^{\prime}$ everywhere in the fluid. At time $t^{\prime}>0$, heat supplied from the cone surface to the fluid and concentration level near the cone surface are raised at a rate of $q_{w}(x)=x^{m}$ and $q_{w}^{*}(x)=x^{n}$, respectively, and they are maintained at the same level. It is assumed that the concentration $C^{\prime}$ of the diffusing species in the binary mixture is very less in comparison to the other chemical species, which are present and hence the Soret and Dufour effects are negligible. We consider viscous flow where pressure work, viscous dissipation, and thermal dispersion effects are neglected. The coordinate system chosen (as shown in Figure 1) is such that the $x$-direction is measured along the cone surface from the leading edge $O$, and the $y$-direction is normal to the cone generator. The cone apex is located at the origin $(x=y=0)$.

Here $\phi$ designates the semivertical angle of the cone, and $r$ is the local radius of the cone.

Then under the previous assumptions, the governing boundary layer equations with Boussinesq's approximation are

$$
\begin{gathered}
\frac{\partial(u r)}{\partial x}+\frac{\partial(v r)}{\partial y}=0 \\
\frac{\partial u}{\partial t^{\prime}}+u \frac{\partial u}{\partial x}+v \frac{\partial u}{\partial y} \\
=v \frac{\partial^{2} u}{\partial y^{2}}-\frac{\sigma B_{0}^{2}}{\rho} u+g \beta \cos \phi\left(T^{\prime}-T_{\infty}^{\prime}\right) \\
+g \beta^{*} \cos \phi\left(C^{\prime}-C_{\infty}^{\prime}\right)-\frac{v}{K} u-\frac{b}{K} u^{2}, \\
\frac{\partial T^{\prime}}{\partial t^{\prime}}+u \frac{\partial T^{\prime}}{\partial x}+v \frac{\partial T^{\prime}}{\partial y}=\alpha \frac{\partial^{2} T^{\prime}}{\partial y^{2}}, \\
\frac{\partial C^{\prime}}{\partial t^{\prime}}+u \frac{\partial C^{\prime}}{\partial x}+v \frac{\partial C^{\prime}}{\partial y}=D \frac{\partial^{2} C^{\prime}}{\partial y^{2}},
\end{gathered}
$$

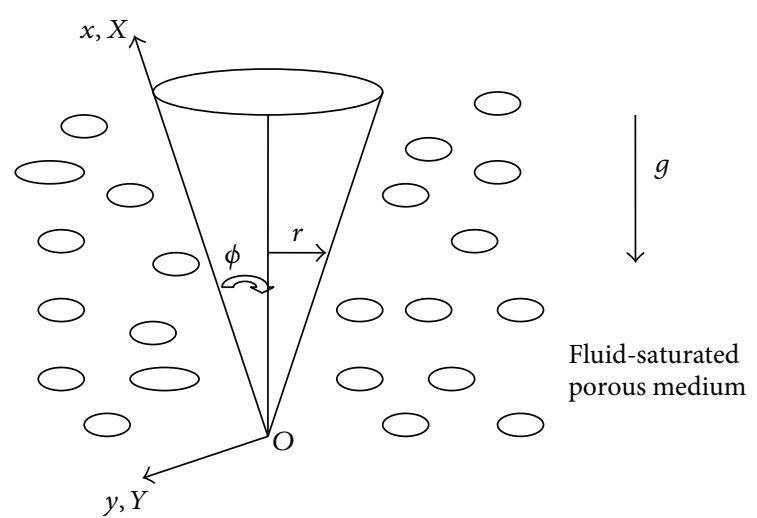

Figure 1: Physical Model.

where all terms are defined in the nomenclature. Under the Boussinesq approximation, buoyancy effects are simulated only in the momentum equation, which is coupled to the energy equation, constituting a free convection regime. The corresponding spatial and temporal initial and boundary conditions at the surface and far from the cone take the following form:

$$
\begin{gathered}
t^{\prime} \leq 0: u=0, \quad v=0, \quad T^{\prime}=T_{\infty}^{\prime}, \quad C^{\prime}=C_{\infty}^{\prime} \forall x, y, \\
t^{\prime}>0: u=0, \quad v=0, \quad \frac{\partial T^{\prime}}{\partial y}=-\frac{q_{w}(x)}{k}, \\
\frac{\partial C^{\prime}}{\partial y}=-\frac{q_{w}^{*}(x)}{D} \quad \text { at } y=0, \\
u=0, \quad T^{\prime}=T_{\infty}^{\prime}, \quad C^{\prime}=C_{\infty}^{\prime} \quad \text { at } x=0, \\
u \longrightarrow 0, \quad T^{\prime} \longrightarrow T_{\infty}^{\prime}, \quad C^{\prime} \longrightarrow C_{\infty}^{\prime} \quad \text { as } y \longrightarrow \infty,
\end{gathered}
$$

where all the parameters are defined in the nomenclature.

Equation (1) is highly coupled, parabolic, and nonlinear. An analytical solution is clearly intractable, and in order to facilitate a numerical solution we nondimensionalize the model. Proceeding with the analysis, we now introduce the following transformations:

$$
\begin{gathered}
X=\frac{x}{L}, \quad Y=\frac{y}{L}\left(\mathrm{Gr}_{L}\right)^{1 / 4}, \\
R=\frac{r}{L}, \quad \text { where } r=x \sin \phi, \\
V=\frac{v L}{v}\left(\mathrm{Gr}_{L}\right)^{-1 / 4}, \quad U=\frac{u L}{v}\left(\mathrm{Gr}_{L}\right)^{-1 / 2}, \\
t=\frac{v t^{\prime}}{L^{2}}\left(\mathrm{Gr}_{L}\right)^{1 / 2}, \quad M=\frac{\sigma B_{0}^{2} L^{2}}{\mu} \mathrm{Gr}_{L}^{-1 / 2}, \\
T=\frac{T^{\prime}-T_{\infty}^{\prime}}{\left[q_{w}(L) L / k\right]}, \quad \mathrm{Gr}_{L}=\frac{g \beta \cos \phi\left[q_{w} L / k\right] L^{4}}{v^{2}},
\end{gathered}
$$




$$
\begin{array}{ll}
\operatorname{Pr}=\frac{v}{\alpha}, \quad \mathrm{Da}=\frac{K}{L^{2}}, & C=\frac{C^{\prime}-C_{\infty}^{\prime}}{\left[q_{w}^{*}(L) L / D\right]}, \\
N=\frac{\beta^{*}\left(C_{w}^{\prime}-C_{\infty}^{\prime}\right)}{\beta\left(T_{w}^{\prime}-T_{\infty}^{\prime}\right)}, & \mathrm{Sc}=\frac{v}{D}, \quad \mathrm{Fs}=\frac{b}{L} .
\end{array}
$$

The transport (1) is thereby reduced to the following dimensionless form:

$$
\begin{gathered}
\frac{\partial(U R)}{\partial X}+\frac{\partial(V R)}{\partial Y}=0 \\
\frac{\partial U}{\partial t}+U \frac{\partial U}{\partial X}+V \frac{\partial U}{\partial Y} \\
=\frac{\partial^{2} U}{\partial Y^{2}}-M U+T \cos \phi+N C \cos \phi \\
-\frac{U}{\mathrm{DaGr}}-\frac{\mathrm{Fs}}{\mathrm{Da}} U^{2} \\
\frac{\partial T}{\partial t}+U \frac{\partial T}{\partial X}+V \frac{\partial T}{\partial Y}=\frac{1}{\operatorname{Pr}} \frac{\partial^{2} T}{\partial Y^{2}} \\
\frac{\partial C}{\partial t}+U \frac{\partial C}{\partial X}+V \frac{\partial C}{\partial Y}=\frac{1}{\mathrm{Sc}} \frac{\partial^{2} C}{\partial Y^{2}}
\end{gathered}
$$

The corresponding nondimensional initial and boundary conditions are given by

$$
\begin{gathered}
t \leq 0: U=0, \quad V=0, \\
T=0, \quad C=0 \quad \forall X, Y, \\
t>0: U=0, \quad V=0, \quad \frac{\partial T}{\partial Y}=-X^{m}, \\
\frac{\partial C}{\partial Y}=-X^{n} \quad \text { at } Y=0, \\
U=0, \quad T=0, \quad C=0 \quad \text { at } X=0, \\
U \longrightarrow 0, \quad T \longrightarrow 0, \quad C \longrightarrow 0 \quad \text { as } Y \longrightarrow \infty,
\end{gathered}
$$

where again all the parameters are given in the nomenclature. The dimensionless local values of the skin friction, Nusselt number, and the Sherwood number are given by the following expressions:

$$
\begin{aligned}
\tau_{x} & =-\left(\frac{\partial U}{\partial Y}\right)_{Y=0} \\
\mathrm{Nu}_{x} & =-X\left(\frac{\partial T}{\partial Y}\right)_{Y=0} \\
\mathrm{Sh}_{x} & =-X\left(\frac{\partial C}{\partial Y}\right)_{Y=0} .
\end{aligned}
$$

\section{Numerical Solution}

In order to solve the unsteady, nonlinear, coupled (4) under condition (5), an implicit finite difference scheme of CrankNicolson type has been employed which is discussed by many researchers [12-15]. The finite difference scheme of dimensionless governing equations is reduced to tridiagonal system of equations and is solved by Thomas algorithm as discussed elsewhere [16]. The region of integration is considered as a rectangle with $X_{\max }=1$ and $Y_{\max }=22$ where $Y_{\max }$ and corresponds to $Y=\infty$ which lies very well outside both the momentum and thermal boundary layers. The maximum of $Y$ was chosen as 22, after some preliminary investigation so that the last two boundary conditions of (5) are satisfied within the tolerance limit $10^{-5}$. The mesh sizes have been fixed as $\Delta X=0.05, \Delta Y=0.05$ with time step $\Delta t=$ 0.01 . The computations are carried out first by reducing the spatial mesh sizes by $50 \%$ in one direction, and later in both directions by $50 \%$. The results are compared. It is observed in all cases that the results differ only in the fifth decimal place. Hence, the choice of the mesh sizes seems to be appropriate. The scheme is unconditionally stable. The local truncation error is $O\left(\Delta t^{2}+\Delta Y^{2}+\Delta X\right)$, and it tends to zero as $\Delta t, \Delta X$ and $\Delta Y$ tend to zero. Hence, the scheme is compatible. Stability and compatibility ensure the convergence. The derivatives involved in (6) are evaluated using five point approximation formula.

\section{Results and Discussion}

Only selective figures have been reproduced here for brevity. In the numerical computations, the following values for the dimensionless thermophysical parameters are prescribed: Grashof number $\left(\mathrm{Gr}_{L}\right)=1.0$, Darcy number $(\mathrm{Da})=0.1$ (high permeability), Forchheimer number (Fs) $=0.1$ (weak quadratic drag), Prandtl number $(\mathrm{Pr})=7.0$ (water), Schmidt number $(\mathrm{Sc})=0.6$ (oxygen diffusing in air), surface heat flux power law exponent $(m)=0.5$, surface mass flux power law exponent $(n)=0.5$, buoyancy ratio parameter $(N)=1.0$, and semivertical angle of the cone $(\phi)=20^{\circ}$. All graphs therefore correspond to these values unless otherwise indicated. To test the accuracy of the computations, the local shear stress and local Nusselt number computations for the nonporous case are compared with those of Hossain and Paul [9] for a heat flux gradient of $m=0.5$ and $X=1.0$ in the steady state, in Tables 1 and 2, respectively, and are found to be in good agreement.

In Figures 2(a) and 2(b), the influence of Grashof number $\left(\mathrm{Gr}_{L}\right)$ on steady state velocity $(U)$ and temperature $(T)$ distributions with $Y$-coordinate are shown. Free convection, that is, thermal buoyancy effects are analyzed via the Grashof number. For an increasing $\mathrm{Gr}_{L}$ from 0.1 through 1.0, 10.0, 50.0 to 100.0 cooling of the cone by free convection occurs; that is, heat is conducted away from the cone to the surrounding regime.

Figures 3(a) and 3(b) show the effect of Darcy number $(\mathrm{Da})$ on dimensionless velocity $(U)$ and temperature $(T)$ with transformed radial coordinate $(Y)$ close to the leading edge (i.e., cone apex) at $X=1.0$. To study the influence of 


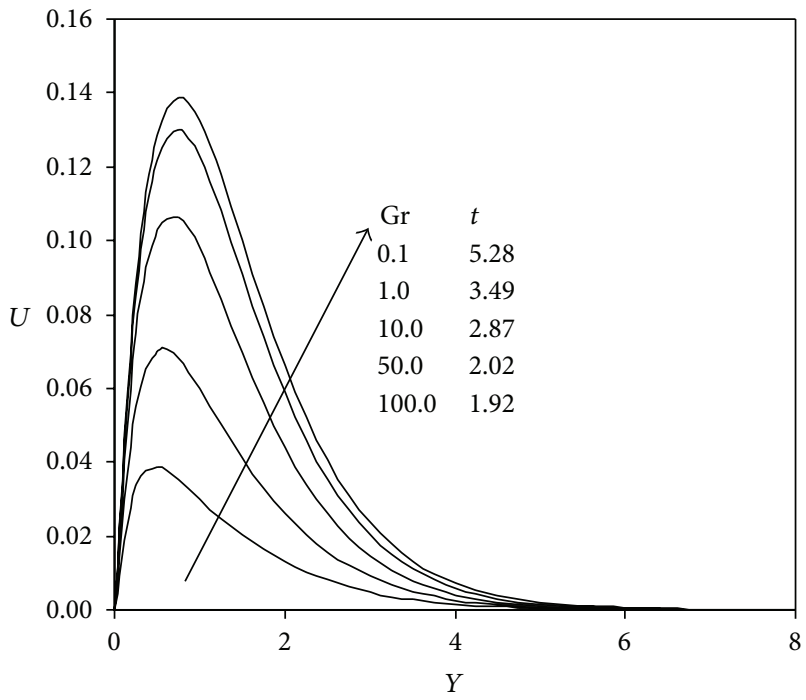

(a)

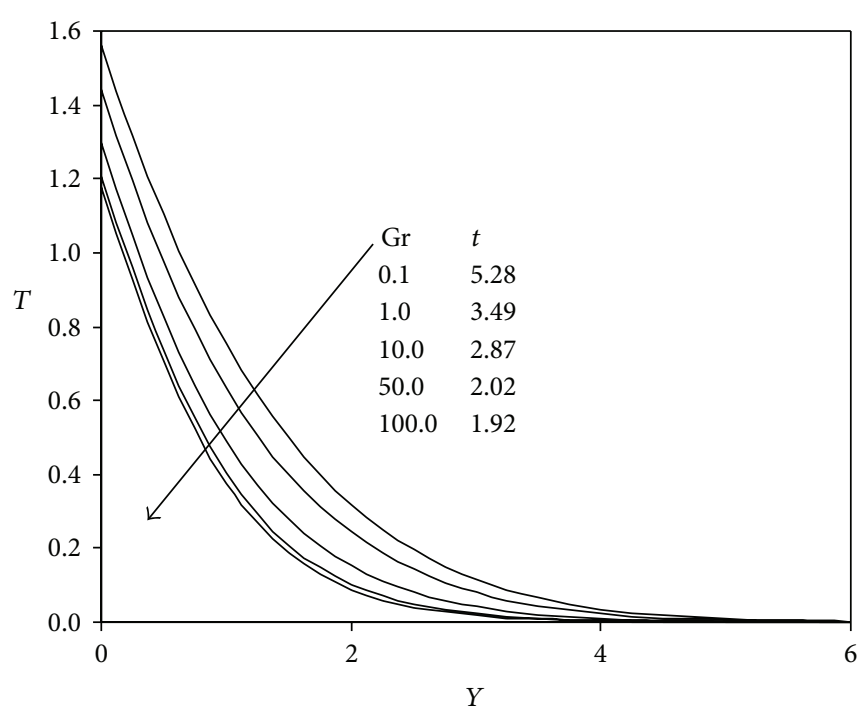

(b)

Figure 2: (a) Steady state velocity profiles at $X=1.0$ for different Gr. (b) Steady state temperature profiles at $X=1.0$ for different Gr.

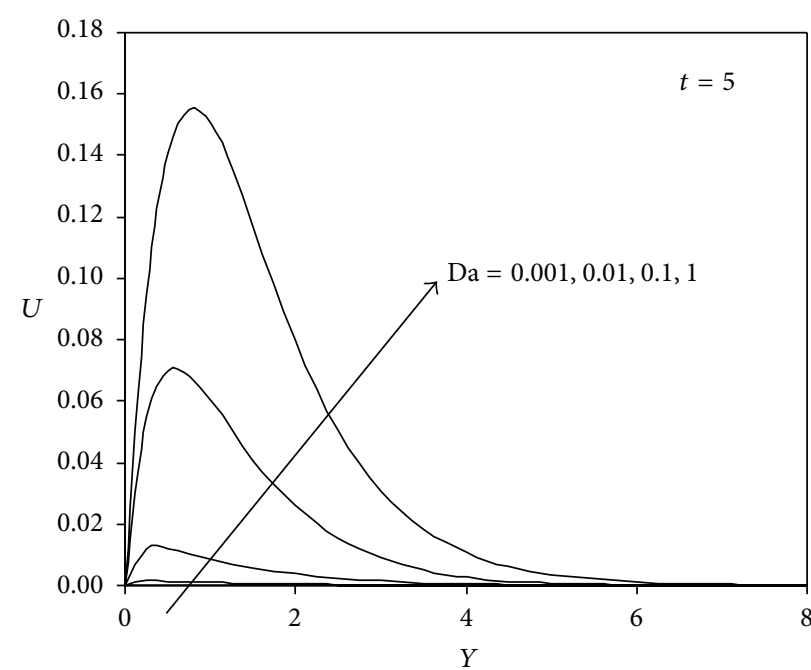

(a)

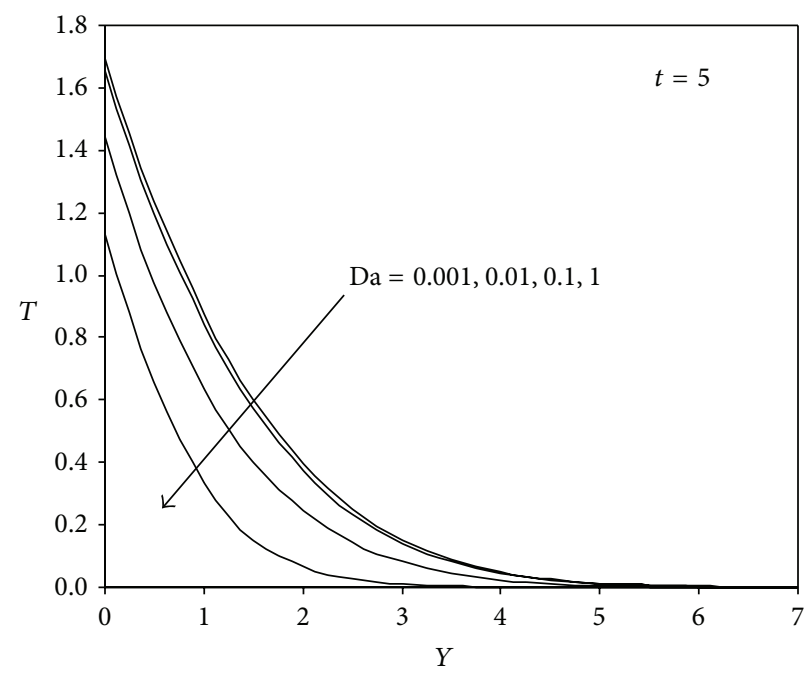

(b)

Figure 3: (a) Transient velocity profiles at $X=1.0$ for different Da. (b) Transient temperature profiles at $X=1.0$ for different Da.

TABLE 1: Comparison of local skin friction values at $X=1.0$ and $m=0.5$ with those of Hossain and Paul [9] for steady state purely fluid ( $\mathrm{Da} \rightarrow \infty$ in present model) case.

\begin{tabular}{lcc}
\hline $\operatorname{Pr}$ & Hossain and Paul [9] $\Gamma_{X} / \mathrm{Gr}_{L}^{3 / 5}$ & Present results \\
\hline 0.01 & 5.13457 & 5.13424 \\
0.05 & 2.93993 & 2.93180 \\
0.1 & 2.29051 & 2.29044 \\
\hline
\end{tabular}

regime permeability from sparsely packed media to densely packed materials, the following values $\mathrm{Da}=1.0,0.1,0.01$, 0.001 are considered. $\mathrm{Da}=K / L^{2}$ for a fixed value of the reference length $(L)$ is directly proportional to permeability
TABLE 2: Comparison of local Nusselt number values at $X=1.0$ and $m=0.5$ with those of Hossain and Paul [9] for steady state purely fluid ( $\mathrm{Da} \rightarrow \infty$ in present model) case.

\begin{tabular}{lcc}
\hline $\operatorname{Pr}$ & Hossain and Paul [9] $\mathrm{Nu}_{X} / \mathrm{Gr}_{L}^{3 / 5}$ & Present results \\
\hline 0.01 & 0.14633 & 0.14648 \\
0.05 & 0.26212 & 0.26227 \\
0.1 & 0.33174 & 0.33648 \\
\hline
\end{tabular}

$(K)$ of the porous regime. Increasing $\mathrm{Da}$ increases the porous medium permeability and simultaneously decreases the Darcian impedance since progressively less solid fibers are present in the regime. The flow is therefore accelerated 


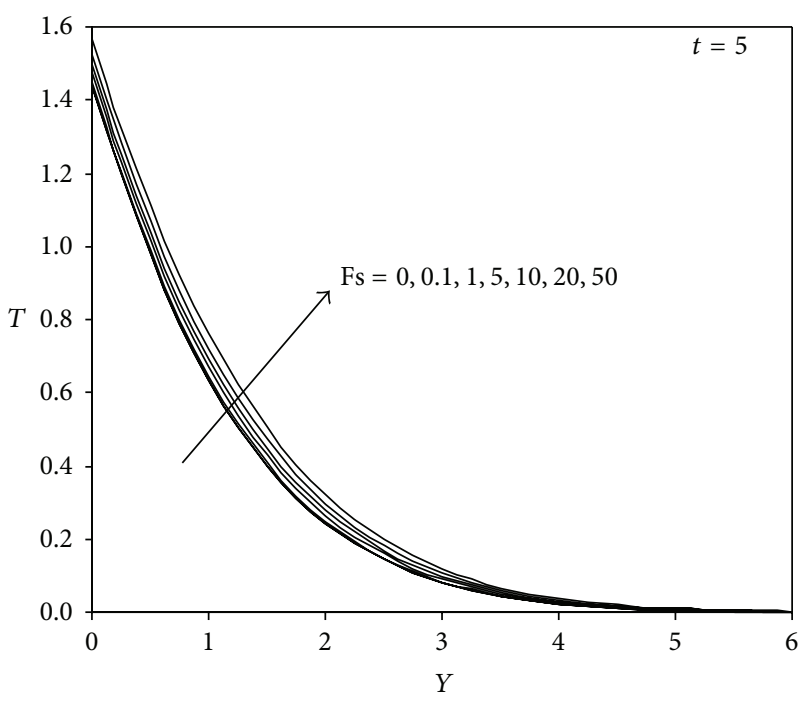

Figure 4: Transient temperature profiles at $X=1.0$ for different Fs.

for higher Da values causing an increase in the velocity $U$ as shown in Figure 3(a). Maximum effect of rising Darcy number is observed at intermediate distance from the cone surface around $Y \sim 1$. Conversely, temperature $T$ depicted in Figure 3(b) is opposed by increasing Darcy number. The presence of fewer solid fibers in the regime with increasing $\mathrm{Da}$ inhibits the thermal conduction in the medium which reduces distribution of thermal energy. The regime is therefore cooled when more fluid is present, and $T$ values in the thermal boundary layer are decreased. Profiles for both velocity and temperature are smoothly asymptotic decays to the free stream indicating that excellent convergence (and stability) is obtained with the numerical method. Velocity boundary layer thickness will be increased with a rise in $\mathrm{Da}$ and thermal boundary layer thickness reduced. The effect of the Forchheimer inertial drag parameter (Fs) on dimensionless temperature $(T)$ profiles is shown in Figure 4. The Forchheimer drag force is a second-order retarding force simulated in the momentum conservation equation. Increasing Fs values from 0.0 through $0.1,1.0,5.0,10.0$, 20.0, and 50.0 causes a strong increase in Forchheimer drag which decelerates the flow, that is, reduces velocities. For higher values of Fs, it is expected that the porous medium flow becomes increasingly chaotic. Temperature $(T)$ however is slightly increased with a rise in Forchheimer parameter. The effects of the Prandtl number (Pr) on velocity profiles are depicted in Figure 5. Pr encapsulates the ratio of momentum diffusivity to thermal diffusivity. Larger Pr values imply a thinner thermal boundary layer thickness and more uniform temperature distributions across the boundary layer. Hence, thermal boundary layer will be much less thick than the hydrodynamic (translational velocity) boundary layer. Smaller Pr fluids have higher thermal conductivities, so that heat can diffuse away from the cone surface faster than for higher Pr fluids (thicker boundary layers). Physically the lower values of $\operatorname{Pr}$ correspond to liquid metals $(\operatorname{Pr} \sim 0.02$, $0.05), \operatorname{Pr}=0.7$ is accurate for air or hydrogen and $\operatorname{Pr}=7.0$

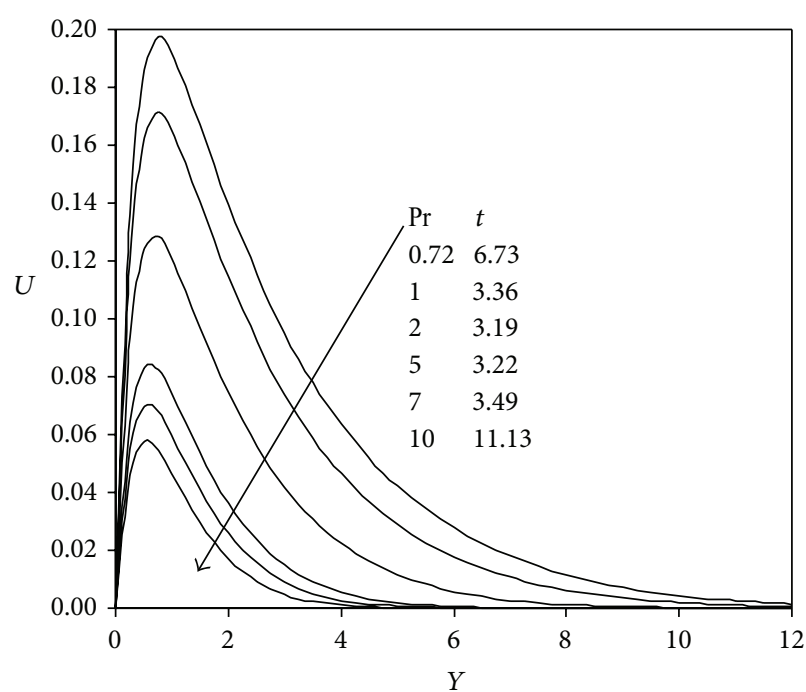

Figure 5: Steady state velocity profiles at $X=1.0$ for different Pr.

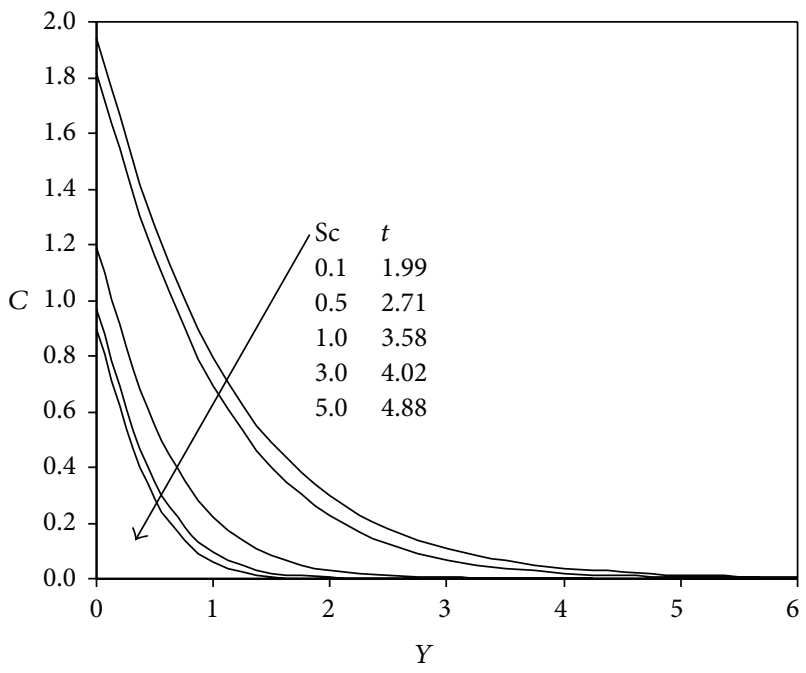

FIGURE 6: Steady state concentration profiles at $X=1.0$ for different Sc.

for water. The computations show that translational velocity $U$ is therefore reduced as $\mathrm{Pr}$ rises from 0.72 through 1.0, 2.0, 5.0, 7.0 and 10.0 since the fluid is increasingly viscous as $\operatorname{Pr}$ rises.

Figure 6 shows the effect of the Schmidt number (Sc) on the dimensionless concentration $(C)$. We note that the Schmidt number (Sc) embodies the ratio of the momentum to the mass diffusivity. Sc therefore quantifies the relative effectiveness of momentum and mass transport by diffusion in the hydrodynamic (velocity) and concentration (species) boundary layers. Smaller Sc values can represent for example hydrogen gas as the species diffusing in air, $\mathrm{Sc}=2.0$ implies hydrocarbon diffusing in air, and higher values to petroleum derivatives diffusing in fluids (e.g. ethyl benzene) as indicated elsewhere [17]. As Sc increases, Figure 6 shows that $C$ values are strongly decreased as larger values of Sc correspond to 


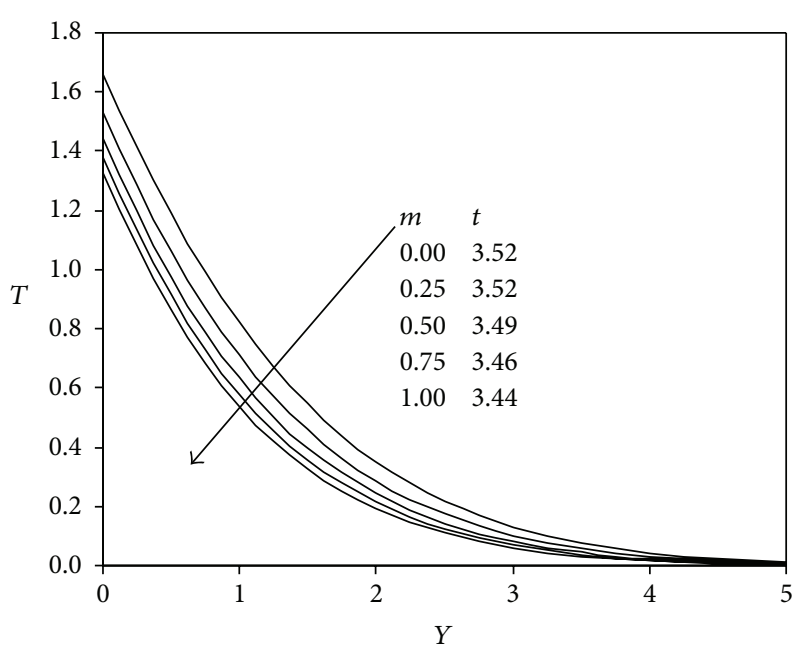

FIgURE 7: Steady state temperature profiles at $X=1.0$ for different $m$.

a decrease in the chemical molecular diffusing that is, less diffusion therefore takes place by mass transport. The dimensionless concentration profiles all decay from a maximum concentration to zero in the freestream. Greater Sc values correspond to lower chemical molecular diffusivity of the parent fluid so that less diffusion of the species occurs in the regime. Concentration boundary layer thickness will therefore be reduced. For low Sc fluid greater species diffusion occurs and concentration boundary layer thickness increased. For $\mathrm{Sc}=1$, the Concentration and velocity boundary layers will have approximately the same thickness that is, species and momentum will be diffused at the same rates. With lower Sc values the decay of concentration from the cone surface is more controlled, for increasing values of Sc the profiles descend more and more steeply and concentration falls faster from the surface to a short distance into the boundary layer regime.

The effect of surface heat flux power exponent $(m)$ on the steady state temperature $(T)$ is shown in Figure 7. An increase in the value of $m$ reduces the temperature. It is also seen that the time required to reach the steady state temperature is more at lower values of $m$. Figure 8 depict the distribution of concentration $(C)$ with radial coordinate $(Y)$ for various values of the surface mass flux power law exponent $(n)$. The concentration reduces with the increasing $n$ values from 0.0 through $0.25,0.50,0.75$ and 1.0. Increasing Fs clearly reduces the local Nusselt number as shown in Figure 9.

A slight increase in local Nusselt number accompanies the increment in $\mathrm{Pr}$ as shown in Figure 10. The influence of the concentration to thermal buoyancy ratio parameter $(N)$, on dimensionless temperature $(T)$ with radial coordinate $(Y)$ is shown in Figure 11. $N=0$ indicates that thermal and species buoyancy forces are both absent. For $N>0$, thermal and species buoyancy forces aid each other. $N=1$ implies that both buoyancy forces are of the same order of magnitude. A rise in $N$ from 0.0 through 1.0, 2.0, 3.0 and 5.0 induces a retarding effect on the flow in the porous regime that is,

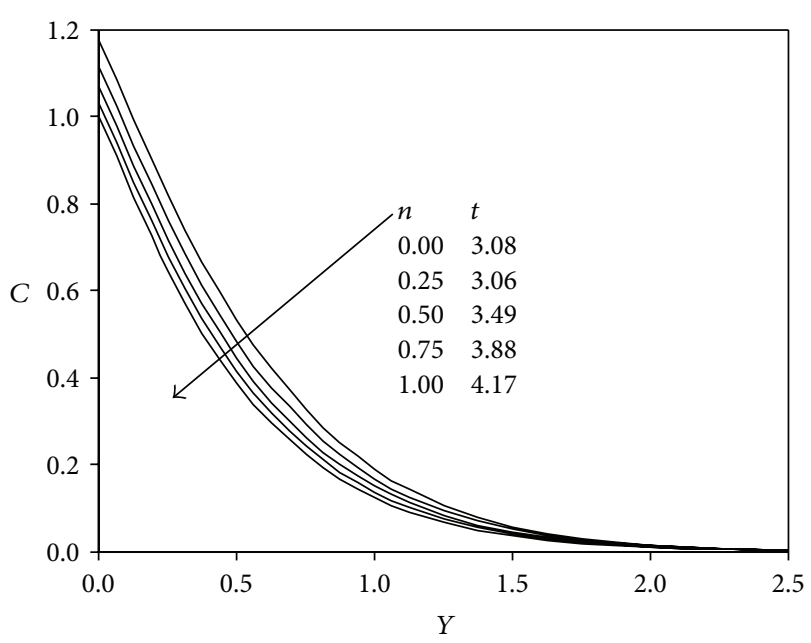

FIGURE 8: Steady state concentration profiles at $X=1.0$ for different $n$.

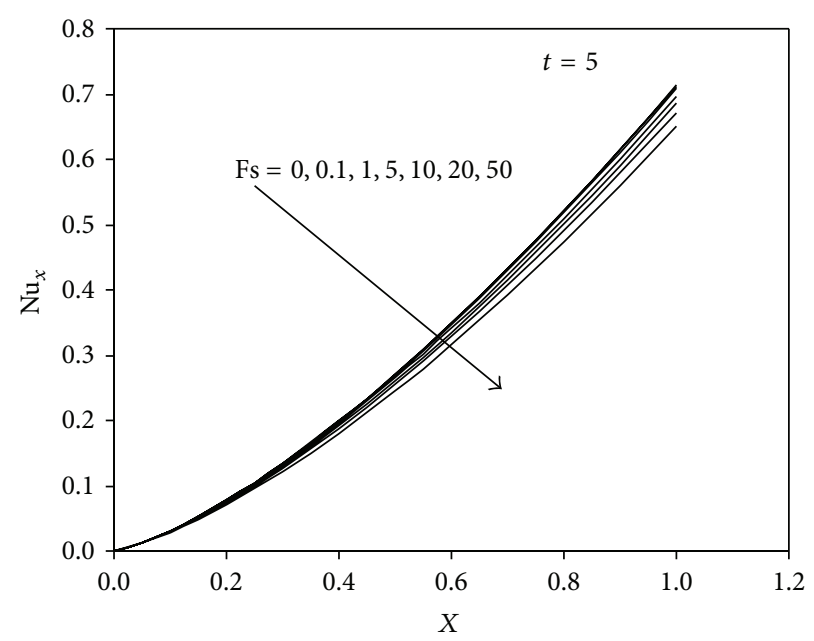

FIGURE 9: Effect of Fs on local Nusselt number at $X=1.0$.

velocities are decreased. Increasing $N$ (thermal and concentration buoyancy forces assisting each other) decreases temperatures in the regime that is, cools the boundary layer regime. The effect of semivertical angle of the cone $(\phi)$ on dimensionless temperature $(T)$ with $Y$-coordinate is shown in Figure 12. It is observed that a rise in $\phi$ substantially increases the temperature $T$ in the boundary layer regime. And more time is required to reach the steady state. Figure 13 the influence of magnetic parameter $(M)$ versus spanwise spatial distributions of velocity $U$ are depicted. Application of magnetic field normal to the flow of an electrically conducting fluid gives rise to a resistive force that acts in the direction opposite to that of the flow. This force is called the Lorentz force. This resistive force tends to slow down the motion of the fluid along the cone and causes an increase in its temperature and a decrease in velocity as $M$ increases. An increase in $M$ from 1 though 2, 3, 4 clearly reduces streamwise velocity $U$ both in the near-wall regime and far-field regime of the boundary layer. 


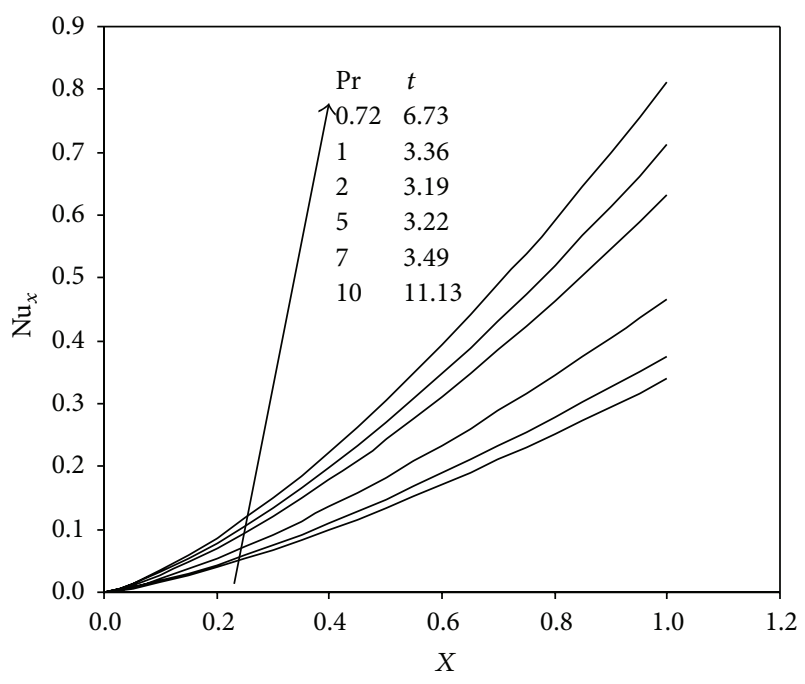

Figure 10: Effect of Pr on local Nusselt number at $X=1.0$.

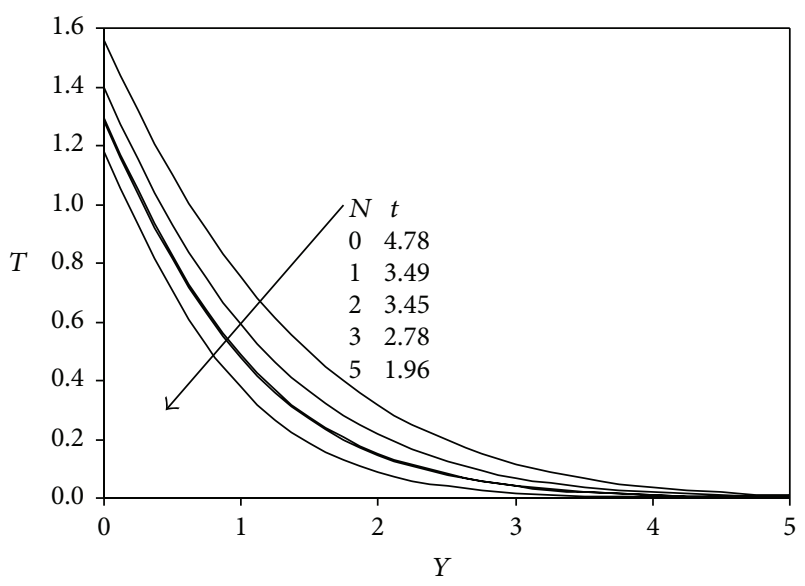

FIGURE 11: Steady state temperature profiles at $X=1.0$ for different $N$.

\section{Conclusions}

Numerical solutions have been presented for the buoyancydriven unsteady natural convection boundary layer flow past a vertical cone embedded in a non-Darcian isotropic porous regime. Present results are compared with those of [8] and found to be in excellent agreement. The following conclusions are drawn.

(i) Increasing Grashof number boosts the translational velocity in the cone surface regime and decreases temperature throughout the flow regime.

(ii) Increasing Darcy number accelerates the flow, that is, increases translational velocities. However, the temperature is reduced with a rise in Darcy number.

(iii) An increase in the Forchheimer inertial drag parameter is observed to slightly increase the temperature but reduces both velocity and local Nusselt number.

(iv) An increase in Prandtl number is observed to decrease both temperature and velocity, but

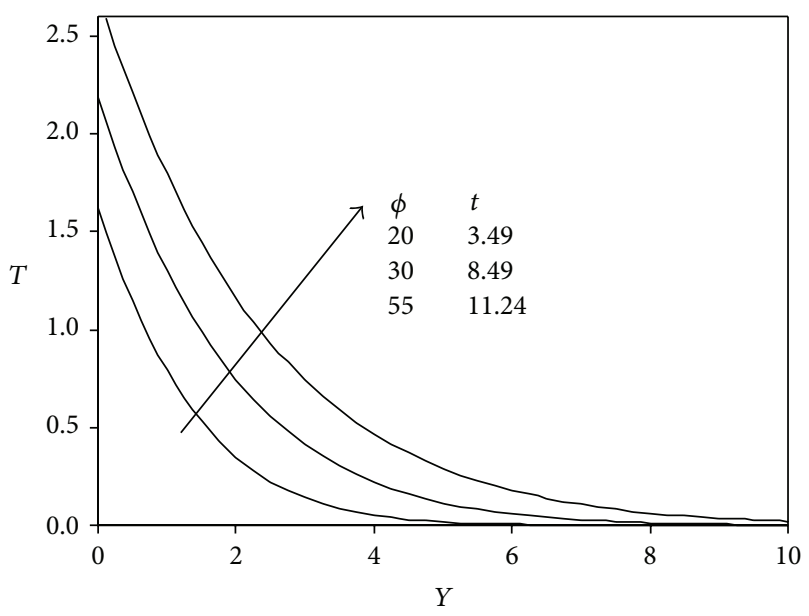

Figure 12: Steady state temperature profiles at $X=1.0$ for different $\phi$.

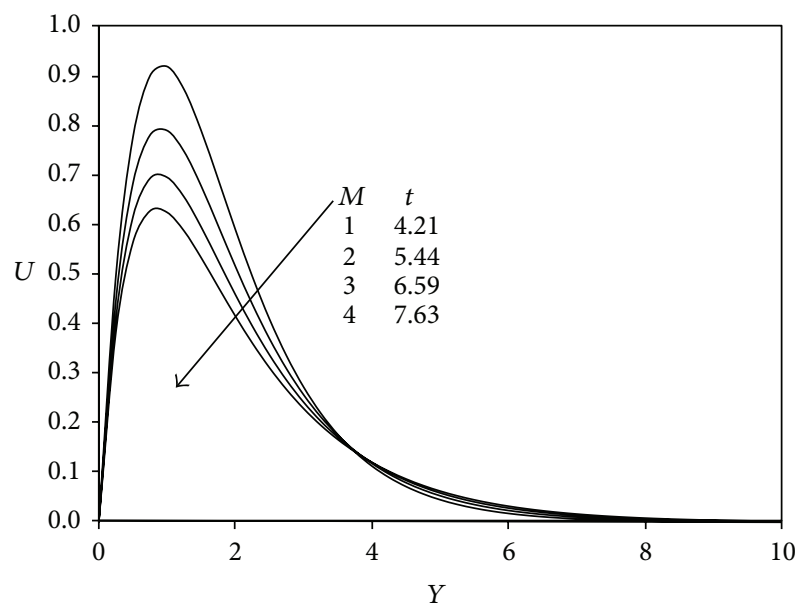

FIGURE 13: Steady state velocity profiles at $X=1.0$ for different values of $M$.

the concentration is slightly increased. A slight increase in local Nusselt number accompanies the increment in Pr.

(v) The concentration is observed to significantly decrease with an increase in Schmidt number.

(vi) The temperature is observed to decrease with an increase in buoyancy ratio parameter but decrease with an increase in semivertical angle of the cone. The time taken to reach the steady state increases with increasing $\phi$.

\section{Nomenclature}

$x, y$ : Coordinates along the cone generator and normal to the generator

$u, v$ : Velocity components along the $x$ - and $y$-directions

g: Gravitational acceleration

$r$ : Local radius of cone 


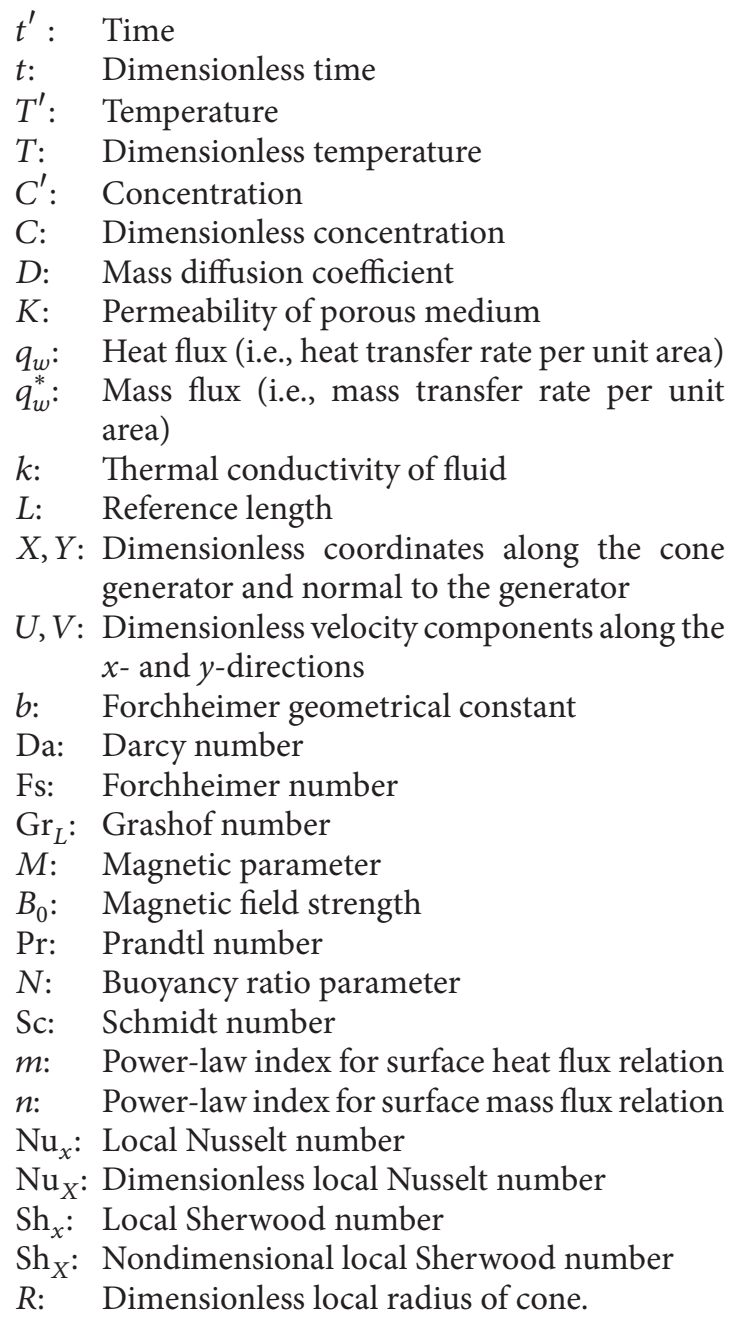

\section{Greek Symbols}

$\mu$ : Dynamic viscosity of fluid

$\nu$ : Kinematic viscosity of fluid

$\phi$ : Semivertical cone angle

$\alpha$ : Thermal diffusivity

$\beta$ : Volumetric thermal expansion coefficient

$\theta$ : Dimensionless temperature function

$\tau$ : Dimensionless time

$\tau_{X}$ : Dimensionless local shear stress function (skin friction).

\section{Subscripts}

$w$ : Condition on the wall

$\infty$ : Free stream condition.

\section{References}

[1] D. B. Ingham and I. I. Pop, Transport Phenomena in Porous Media II, Pergamon Press, Oxford, UK.

[2] D. A. Nield and A. Bejan, Convection in Porous Media, Springer, New York, NY, USA, 3rd edition, 2006.

[3] K. Vafai, Handbook of Porous Media, CRC Press, Boca Raton, Fla, USA, 2nd edition, 2005.
[4] O. V. Trevisan and A. Bejan, "Combined heat and mass transfer by natural convection in a porous medium," Advances in Heat Transfer, vol. 20, pp. 315-352, 1990.

[5] K. Vafai and C. L. Tien, "Boundary and inertia effects on flow and heat transfer in porous media," International Journal of Heat and Mass Transfer, vol. 24, no. 2, pp. 195-203, 1981.

[6] C.-H. Chen and C.-K. Chen, "Non-darcian mixed convection along a vertical plate embedded in a porous medium," Applied Mathematical Modelling, vol. 14, no. 9, pp. 482-488, 1990.

[7] C. K. Chen, C. Chien-Hsin, W. J. Minkowycz, and U. S. Gill, "Non-Darcian effects on mixed convection about a vertical cylinder embedded in a saturated porous medium," International Journal of Heat and Mass Transfer, vol. 35, no. 11, pp. 30413046, 1992.

[8] M. A. Hossain and S. C. Paul, "Free convection from a vertical permeable circular cone with non-uniform surface temperature," Acta Mechanica, vol. 151, no. 1-2, pp. 103-114, 2001.

[9] M. A. Hossain and S. C. Paul, "Free convection from a vertical permeable circular cone with non-uniform surface heat flux," Heat and Mass Transfer, vol. 37, no. 2-3, pp. 167-173, 2001.

[10] A. J. Chamkha, A.-R. A. Khaled, and O. Al-Hawaj, "Simultaneous heat and mass transfer by natural convection from a cone and a wedge in porous media," Journal of Porous Media, vol. 3, no. 2, pp. 155-164, 2000.

[11] K. Vajravelu and J. Nayfeh, "Hydromagnetic convection at a cone and a wedge," International Communications in Heat and Mass Transfer, vol. 19, no. 5, pp. 701-710, 1992.

[12] R. Muthukumaraswamy and P. Ganesan, "Unsteady flow past an impulsively started vertical plate with heat and mass transfer," Heat and Mass Transfer, vol. 34, no. 2-3, pp. 187-193, 1998.

[13] S. Gouse, Mohiddin Computational Fluid Dynamics, LAP Lambert Academic Publishing, Saarbrücken, Germany, 2011.

[14] S. G. Mohiddin, V. R. Prasad, S. V. K. Varma, and O. Anwar Bég, "Numericalstudy of unsteady free convective heat and mass transfer in a walters-b viscoelastic flow along a vertical cone," International Journal of Applied Mathematics and Mechanics, vol. 6, pp. 88-114, 2010.

[15] P. Bapuji, K. Ekambavanan, and A. J. Chamkha, "Unsteady laminar free convection from a vertical cone with uniform surface heat flux," Nonlinear Analysis, vol. 13, pp. 47-60, 2008.

[16] B. Carnahan, H. A. Luther, and J. O. Wilkes, Applied Numerical Methods, John Wiley \& Sons, New York, NY, USA, 1969.

[17] B. Gebhart, Y. Jaluria, R. L. Mahajan, and B. Sammakia, Buoyancy-Induced Flows and Transport, Hemisphere, New York, NY, USA, 1998. 

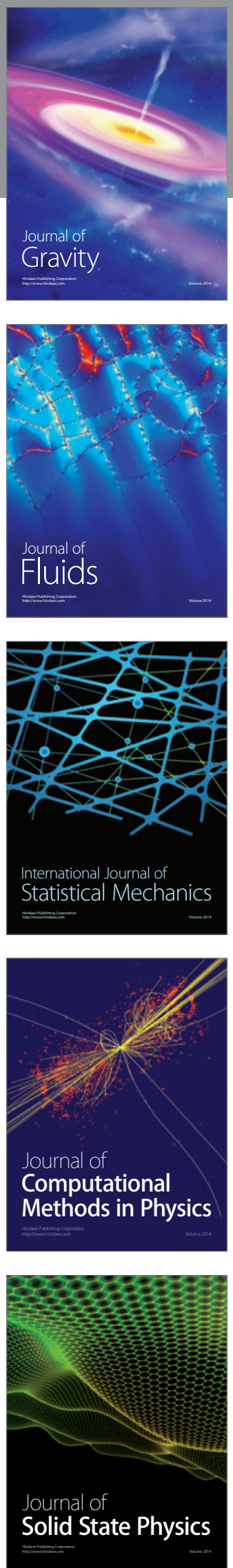

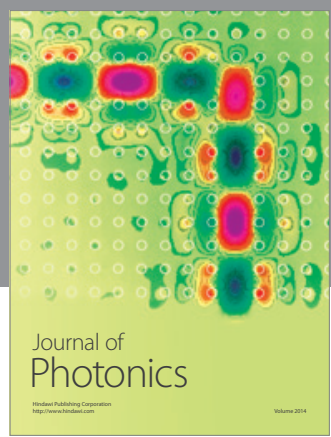

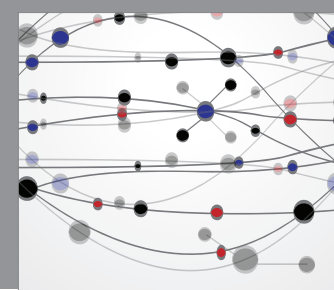

The Scientific World Journal

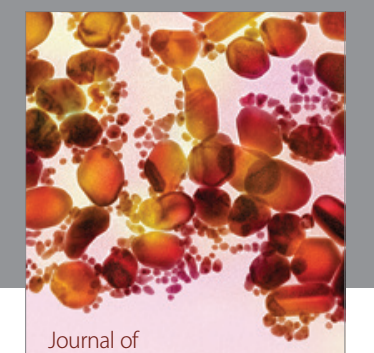

Soft Matter
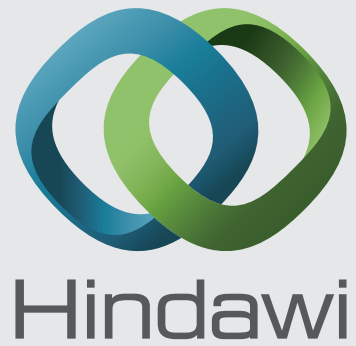

Submit your manuscripts at

http://www.hindawi.com
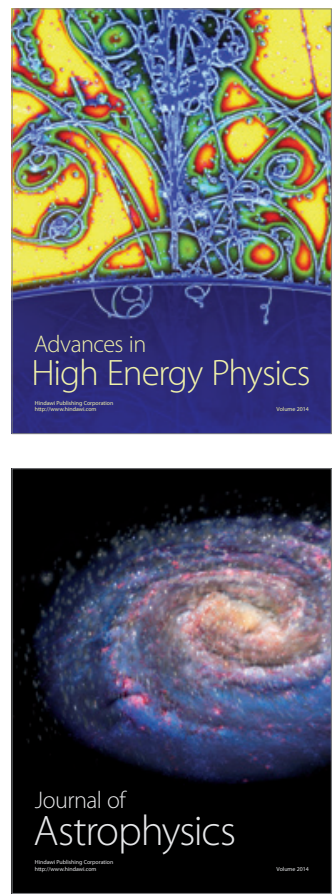
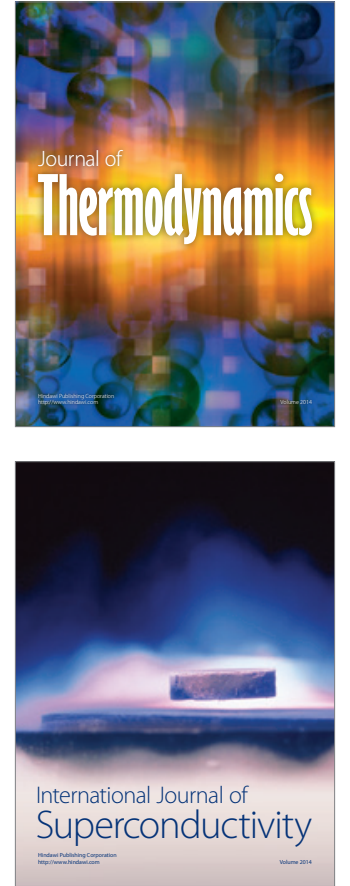
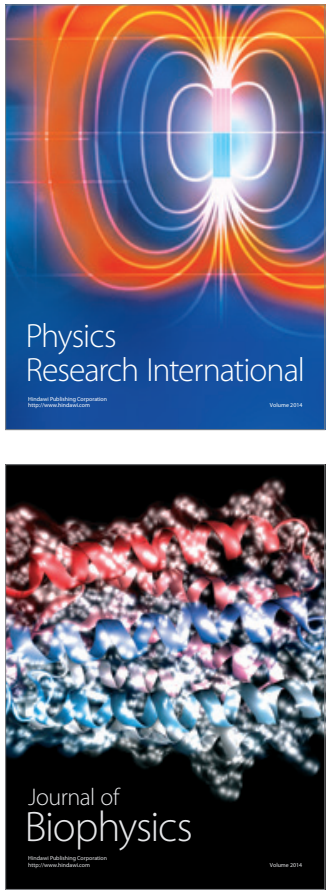
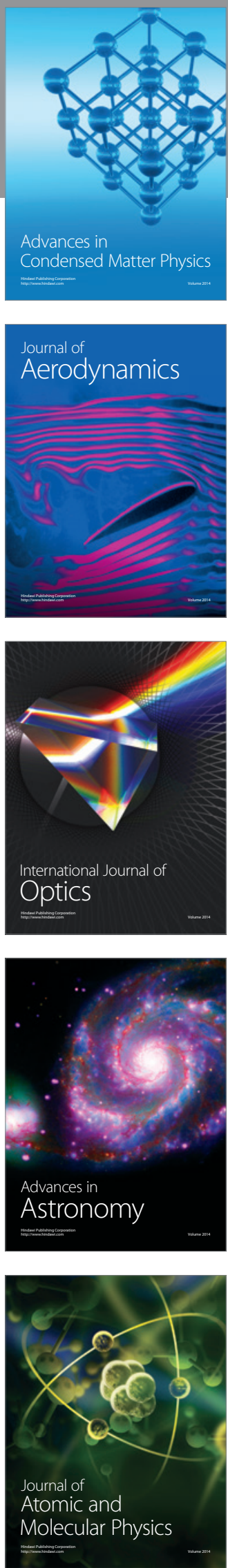